\title{
Layered Metals Fabrication Technology Development for Support of Lunar Exploration at NASA/MSFC
}

\author{
Kenneth G. Cooper ${ }^{1}$, James E. Good ${ }^{2}$, Scott D. Gilley ${ }^{3}$ \\ 'NASA Marshall Space Flight Center, Huntsville, AL 35812, USA \\ ${ }^{2}$ MEI Technologies, Inc., Huntsville, AL 35805 \\ ${ }^{3}$ Tec-Masters, Inc., Huntsville, AL 35805 \\ 256-544-8591; Kenneth.G.Cooper@nasa.gov
}

\begin{abstract}
NASA's human exploration initiative poses great opportunity and risk for missions to the Moon and beyond. In support of these missions, engineers and scientists at the Marshall Space Flight Center are developing technologies for ground-based and in-situ fabrication capabilities utilizing provisioned and locally-refined materials. Development efforts are pushing state-of-the art fabrication technologies to support habitat structure development, tools and mechanical part fabrication, as well as repair and replacement of ground support and space mission hardware such as life support items, launch vehicle components and crew exercise equipment. This paper addresses current fabrication technologies relative to meeting targeted capabilities, near term advancement goals, and process certification of fabrication methods.
\end{abstract}

Keywords: Layered fabrication, solid freeform fabrication, rapid manufacturing, additive fabrication. PACS: 81.05.Bx, 81.15.Jj, 81.05.Mh

\section{INTRODUCTION}

A Fabrication Technologies research and hardware development program for support of space missions is underway at the NASA Marshall Space Flight Center (MSFC) in Huntsville, Alabama. This effort is a sub-element of the In Situ Fabrication and Repair (ISFR) element, which is working in conjunction with the In Situ Resources Utilization (ISRU) element. ISFR has the charter to provide fabrication and repair capabilities for space mission equipment with long term goal of operation in extraterrestrial environments while ISRU will extract useable materials from those local environments. ISFR activities include fabrication of mechanical components and assemblies on Earth as well as within partial gravity environments with optional consideration of micro-gravity operations capability for intransit phases. The effort includes development of supporting fabrication, repair, and habitat structure technologies to maximize use of in situ resources. However, provisioned fabrication feedstocks may be launched with the carrier vehicle and utilized either during in-transit phases or in situ until ISRU product feedstocks are available.

The current research effort has some past history in that layer-by-layer manufacturing has been investigated for spaceflight endeavors. The level of maturity of this technology as recent as a few years ago left a large gap between state-of-the-art additive manufacturing techniques before and what is achievable currently. Although this is true, MSFC was looking ahead (Cooper, 2001) in the 1990s and investigated the possibility of microgravity manufacturing using additive fabrication techniques. Additionally, applications in outer space (Cooper, 2002) were investigated and these efforts provided a foundation for the current development program. Significant rapid manufacturing techniques and material development advancements have been made in the last two years and require a serious investigation to determine the feasibility of in situ manufacturing for space applications and to determine technology gaps. 
The ISFR Element supports the entire life cycle of the Exploration program by: reducing downtime due to failed components; decreasing risk to crew by recovering quickly from degraded operation equipment; improving system functionality with advanced geometry capabilities; and enhancing mission safety by reducing assembly part counts of original designs where possible. Non-destructive Evaluation (NDE) capabilities will help reduce crew exposure to harsh space environments by providing autonomous technologies capable of identifying and confirming a failure or validating that a repair or fabrication operation was successful (Bodiford, 2005). NDE will also be incorporated within fabrication systems where possible to verify integrity, material properties and dimensional accuracy during the build cycle.

ISFR also provides habitat manufacturing and assembly technologies that incorporate in situ resources to produce autonomous, affordable, and pre-positioned habitat environments. These habitats will feature radiation shielding and protection from micrometeoroids and exhaust plumes. ISFR strives to reduce launched mass and volume resource requirements for supply of spares and materials from Earth by utilizing in situ resources. In addition, ISFR is investigating just-in-time repair capability using soldering, patching, and adhesives to return functionality to components that may be repairable rather than require complete replacement.

This paper has been published in an effort to provide the current technologies available for direct metal fabrication with a specific emphasis on the Arcam EBM process. Significant advances have allowed the gap to close between rapid prototypes and rapid manufacturing. These current processes can provide an important capability on the lunar surface while minimizing the upmass of traditional feedstock. These are crucial components for a successful longduration mission to the moon.

\section{FABRICATION TECHNOLOGIES OVERVIEW}

This article specifically focuses on fabrication of metal components intended to initially produce flight hardware on Earth prior to mission launch as well as on-demand replacement of failed or new components in situ. Earth-based fabrication is targeted at providing rapid turn around of prototypes production as well as future flight components with complex geometries that are difficult or impossible to fabricate with traditional machining, casting or forming methods. Future in situ fabrication goals include creation of replacement components and unforeseen tools that may enhance mission success as well as correction of design deficiencies during the mission for which spares are of little use if failures occur.

The main goal of Fabrication Technologies will be to provide rapid manufacture of parts and tools via a qualitycontrolled approach that may be a single process or a hybrid mix of additive and subtractive processes. An important near term benefit will be the production of advanced geometry components and assemblies on Earth prior to launch as part of the mission equipment design. Earth-based component production activities will serve to provide immediate benefits of the development investment that may lead to future in situ fabrication operations. Development demonstrations will include production of components for functional testing with certification of the fabrication processes to produce pedigreed components.

\section{Fabrication Processes Discussion}

An initial study of fabrication technologies performed at MSFC showed that there is no single comprehensive solution for fabrication systems in space (Hammond, 2006). A majority of space-flight hardware is fabricated using typical subtractive processes such as Computer Numerically Controlled (CNC) machining that provide excellent dimensional accuracy and surface finish. However, they require feedstock material that is larger than the outer part envelope and also usually require part-specific hold down tooling that is not very mass efficient for complex geometry components. Machining processes also typically require bulky and heavy equipment to provide a stable platform for significant material removal operations. An anticipated approach will involve a combination of additive and subtractive technologies to provide the necessary fabrication capabilities.

Additive techniques typically involve building parts layer-by-layer using filaments, powders, liquids or stacked sheets of feedstock materials successively joined together to build up a part in three dimensions. These methods offer reduction of feedstock requirements since parts are built up to size from a common feedstock size rather than subtractively machined down from bulk stock that must be larger than the part envelope. Current additive 
limitations include reduced accuracy and surface finish compared to traditional machining. Incorporating additive methods with light $\mathrm{CNC}$ machining processes integral to the system design is currently envisioned to provide required accuracies and feedstock efficiency. This also supports machining of certified feedstocks in situations where additive techniques may not be able to produce acceptable material properties by a constrained resource fabrication system. These two techniques, functioning in tandem, would thus provide improved capability over either single process. Based on a materials utilization study (Hammond, 2006), the initial focus of fabrication technologies development is focusing on metallic components. This paper discusses additive manufacturing technologies in reference to future application for space flight missions.

\section{Materials Set Discussion}

Before evaluating specific additive techniques, the desired material set was first defined (Hammond, 2006). Technical interchange meetings with multiple flight hardware development programs at NASA/MSFC were conducted to determine material needs. Also, a statistical analysis of the materials utilized in several previously flown space-flight hardware projects including the Material Identification and Usage Lists (MIULs) of Space Station and Shuttle Mid-Deck Payloads. Another source of information used to determine the material set was a compilation of failure data representing selected space vehicles. This list contained failed components as well as the component material. The list provided insight into the type of components that may require replacement, as well as the type of components requiring fabrication during a long-duration stay on the Moon or Mars. The primary set of metallic materials was thereby identified as shown in Table 1.

TABLE 1. Primary Metallic Materials Set Targeted for Fabrication Technologies Development.

\begin{tabular}{|l|l|}
\hline MATERIAL & ALLOY \\
\hline Aluminum & 6061 \\
\hline Aluminum & 7075 \\
\hline Titanium & Ti6Al4V \\
\hline Stainless Steel & 316 \\
\hline Stainless Steel & $17-4 \mathrm{PH}$ \\
\hline Inconel & 625 \\
\hline Inconel & 718 \\
\hline
\end{tabular}

\section{Additive Fabrication Processes Assessment}

A trade study was performed to investigate additive techniques that could manufacture metal parts in support of space-flight missions. The systems selected for evaluation included the following technologies and assessed for their metal fabrication capabilities and the ability to process the desired materials. Also included in the assessment were part accuracy, surface finish, feedstock type, feedstock usage efficiency, and part build volume among other factors. The systems selected for evaluation are shown in Table 2 . These systems produce parts by consolidating feedstock one layer at a time formed to shape the part cross section geometry at that particular build height.

TABLE 2. Assessed Additive Fabrication Processes.

\begin{tabular}{|l|l|}
\hline SYSTEM OR METHOD & DESCRIPTION \\
\hline Selective Laser Sintering (SLS(B)-EOS® & $\begin{array}{l}\text { Sinters successively spread layers of powder in raster fashion } \\
\text { using laser beam. }\end{array}$ \\
\hline Arcam Electron Beam Melting (EBM) & $\begin{array}{l}\text { Fuses successively spread layers of powder in raster fashion } \\
\text { using electron beam. }\end{array}$ \\
\hline Laser Engineered Net Shaping (LENS®) & $\begin{array}{l}\text { Fuses flowing powder sprayed by gas nozzle into laser beam to } \\
\text { build parts progressively in vertical direction. }\end{array}$ \\
\hline Prometal 3DP & $\begin{array}{l}\text { Consolidates successively spread layers of powder by adhesive } \\
\text { spray will follow-up sintering and infiltration to densify parts. }\end{array}$ \\
\hline POM Group - Direct Metal Deposition (DMD®) & $\begin{array}{l}\text { Fuses flowing powder sprayed by gas nozzle into laser beam to } \\
\text { build parts progressively in vertical direction. }\end{array}$ \\
\hline SLS(®)-3D SYSTEMS & $\begin{array}{l}\text { Sinters successively spread layers of powder in raster fashion } \\
\text { using laser beam. Requires infiltration for densification. }\end{array}$ \\
\hline Ultrasonic Consolidation (UC) & $\begin{array}{l}\text { Consolidates successively applied metal tape using ultrasonic } \\
\text { welding with integral CNC functions to machine to net shape. }\end{array}$ \\
\hline
\end{tabular}


The process trade study focused on two primary areas: the part quality and material set. A $50 \%$ weighting was placed on the quality of the part, including capabilities of the system with respect to the part build volume, geometrical tolerances, and surface finish among others. Relative rankings were then assigned to each system for all part quality criteria. A $50 \%$ weighting was also placed on the system ability to process a set of 14 typical aerospace materials, including the primary set listed in Table 1 . The evaluation was performed by members of the Fabrication Technologies team along with the members of MSFC Rapid Prototyping Laboratory. Two processes that distinguished themselves based on current technological maturity and NASA requirements were Electron Beam Melting (EBM) and Selective Laser Sintering (SLS). These two processes scored very closely in the trade study on their overall weighted score total with individual strengths and weaknesses taken into account. The EBM score was slightly higher and was chosen for further in-house development. Deciding factors included the EBM metallic materials set better matches those desired, it produces fully dense parts and build operations are performed in a vacuum chamber, which fits well with the space environment. The trade off with EBM is that it has a rough surface finish with lower absolute accuracy but these can be corrected via post processing with $\mathrm{CNC}$ machining, which is envisioned for an integrated system anyway.

\section{The Electron Beam Melting (EBM) Technology}

The EBM technology was chosen for further in-house development due to its ability to produce a fully-dense metal material that yields high strength properties (Lindhe, 2003). While surface finish is not at the level desired by many industries or customers, this can be addressed through secondary processes such as CNC machining. Many components used in the aerospace field do not require a polished finish as a requirement, but merely is a desire. As this technology becomes established as a viable solution to component manufacturing, the designers must change the way these components are designed.

There are similarities between the EBM process and the SLS process in that both processes use a power source to heat a bed of powder layer-by-layer to additively "grow" a part. The main difference being the source of the power required to change the metal powder to a finished part. The laser has been used in the rapid prototyping industry for years and is the backbone for the SLS process. The electron beam gun is used in the Arcam machine to fully melt the metal powder in the EBM process. The electron beam has shown several advantages over the laser and its popularity is increasing as the early adopters have had success in part-fabrication and material characterization, validating the claims of the vendor that the EBM machine can produce fully dense material. The efficiency of the electron beam is five to 10 times more energy efficient than laser technology. This is important as it results in less power consumption and lower maintenance and manufacturing costs. Arcam is using a 4000 Watt electron beam gun in their current EBM machines. It is this power that results in the full melt of the powder that allows for good material properties at high build speeds.

The EBM process starts with the pre-heating of the powder bed. The $4 \mathrm{~kW}$ electron beam gun is used to perform the pre-heat by using a low beam current but a high scan speed. The layers are heated after the initial .1 mm layer is spread across the bed. The scan speed can be as high as 1000 meters $/ \mathrm{sec}$ with a build rate up to $60.6 \mathrm{~cm}^{3} / \mathrm{hour}$. The preheat is performed for two reasons:

- The preheat lightly sinters the metal powder to hold it in place for the subsequent layers used to fabricate the part.

- The preheat provides heat to the fabricated part during the build which reduces the thermal gradient between the last melted layer and the previously melted layers. The consistent temperature of the build will reduce the chance of residual stresses.

How does the beam get to the powder bed? The beam is generated by the electron beam gun which is fixed to the top of the vacuum chamber. The beam is deflected to reach the entire build volume. The deflection is achieved by the set of 2 magnetic coils. The first magnetic coil acts as a lens and focuses the beam to the desired diameter. The second magnetic coil deflects the beam to the desired location on the powder bed. Take note that no moving parts are needed to deflect the beam. The beam is created by heating a tungsten filament to a high temperature where many electrons are accelerated to half the speed of light and stream through the gun. After proceeding through the magnetic coils, the beam begins melting each layer of the fabricated part. 
After each layer is melted, the build platform lowers the amount equal to one layer-thickness. A rake spreads the powder and is then melted. This pattern continues until the part has been completed. At this point a helium purge is initiated to minimize the cool-down time. Without the purge, the part may require in excess of 20 hours to cool while the addition of the helium purge reduces the wait time to between 3-8 hours depending upon the part size. A completed part that has a large dimension in the Z-direction will require a longer time to cool the vat of powder because of the depth. A good scenario is one in which the part, or parts, is completed at the end of the day and can cool down overnight. Prior to taking the part out of the chamber, air is purged into the chamber to equalize the pressure to that of the exterior environment. At this point, the door is opened and the loose powder is removed from the part, it is bead-blasted to remove any stubborn powder attached to the surface. The bead blast is performed with the same material as the finished part. The part is now ready for post-processing of any critical interfaces, such as machining a good surface at a mated interface.

This is a good time to note that the finished part is fully dense and does not require a secondary process where it is infiltrated to reduce porosity. Again, this is the major difference between the EBM and SLS processes. A characteristic of both processes is the ability to produce unique geometries such as internal cavities that are used as conformal cooling channels. These cooling channels cannot be produced easily from conventional CNC machining. The EBM process can fabricate a part that contains a matrix core with a solid shell, effectively having a hollow part supported with an internal scaffold. This is a situation where CNC machining is not an option. A similar part is shown in Figure 1 where a scaffold is integrated within the four chambers of the flanged component. The scaffold provides a flow path where surface area is a critical component. It provides high surface area and low pressure drop through the matrix. This is advantageous for the purpose of this component which involves a life support system performing in a space environment.

The propulsion group at NASA/MSFC has been investigating the EBM process for parts fabrication. Turbomachinery components have been made (see Figure 2) to be used in functional testing alongside similar parts made by traditional methods. Tests are on-going and results are not available at this time but with the ability to redesign the components and fabricate the parts quickly, the EBM process can speed up the design process.

As a demonstration part for the Crew Launch Vehicle (CLV), the Rapid Prototyping Lab at MSFC worked with the CLV design team to look for situations where the EBM process could aid the design. A titanium end fitting (see Figure 3) was chosen and fabricated in 8 hours in order to demonstrate the quick turn-around time and the capability to fabricate such a labor-intensive part. Typically, these end fittings, that were part of the initial design of the Interstage, are machined out of a large block of titanium; however, CNC machining could not access part of the geometry that needed to be eliminated. In the end, the machined part was approximately $.23 \mathrm{Kg}$ heavier than the designed part, where the baseline weight was approximately $1.8 \mathrm{Kg}$. The EBM part was able to build the geometry, as designed, and at the expected weight.

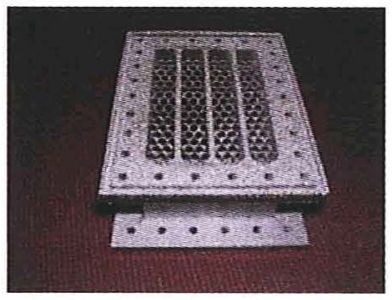

FIGURE 1. ECLSS Component.
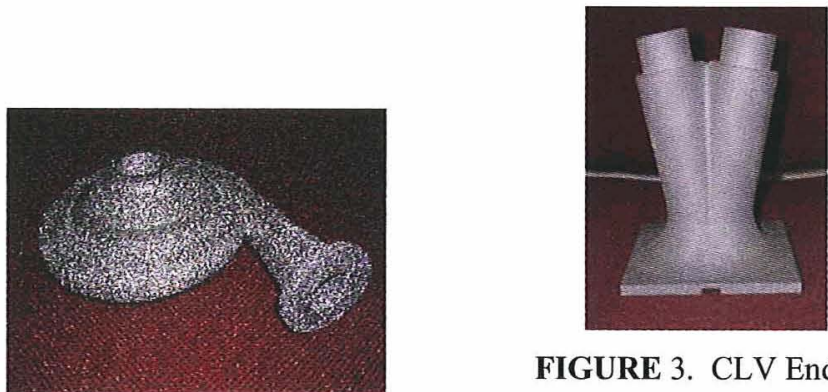

FIGURE 3. CLV End Fitting.

FIGURE 2. Turbopump Component. 


\section{Material Feasibility Studies of Selected Materials}

Marshall Space Flight Center has contracted Arcam to perform feasibility studies on selected materials that are of interest in the aerospace industry, as well as the military. These materials include Inconel 625 and 718 , aluminum 6061, stainless steel 316, and 17-4 steel. Research has shown that these materials have been used extensively in space systems found on the space shuttle, space station, the MIR, and other vehicles. A suite of materials such as these would increase the demand for the EBM process. Currently, the Inconel 625 feasibility study has been completed. The results show the material performs very well in the EBM machine.

Inconel 625 is a nickel-based superalloy well-known for its high temperature and wet corrosion resistance. The study that was performed focused on the melting properties and whether the process was stable over hundreds of melted layers. No actual parts were made and parameter optimization was not a goal of this study.

Two different materials were used in this study and supplied by two independent powder manufacturers. It was seen from the results that the pedigree of the powder can make a difference in the performance of the powder. The figure, shown below, illustrates the cross-section of the powder particles. Impurities can be seen on the left-column samples as well as some spherical porosity.

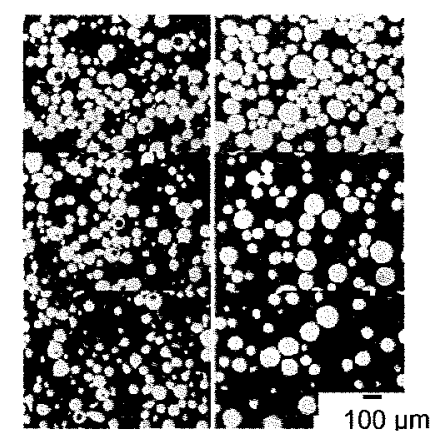

The right column photos show a powder that is almost perfectly spherical and is virtually free from impurities and pores.

The impurities of the one powder source was observed in the melt pool throughout the builds and may be the result of silicon, magnesium, iron and oxygen

The tensile strength was consistent with the impurity levels of the two powders. The powder with the greater level of impurities and porosity reflected lower strengths in all samples tested.

FIGURE 4. Powder Cross-Section.

The sample parts were fabricated using both powders and analyzed. Once again, the best surface quality was obtained from the "better" powder.

These parts were limited in height due to the availability of the powders. The base of the samples measured $15 \times 80$ $\mathrm{mm}$ with a height of $20-30 \mathrm{~mm}$. Because of the limited powder, only horizontal tensile bars were fabricated for testing.

The images at the bottom of Figure 4 show dark particles in the top surface of the samples and are believed to come from impurities in the powder.

Samples were tested in their original state, directly from the EBM machine. No post-build treatments were performed outside of sand blasting to remove excess powder.

In addition to the previously mentioned tensile tests, the samples underwent additional investigations including:

- X-Ray fluorescence (XRF) for metallic content

- High temperature combustion with IR detection for oxygen content

- Portable Brinell hardness tester

- Optical microscopy for microstructure examination 

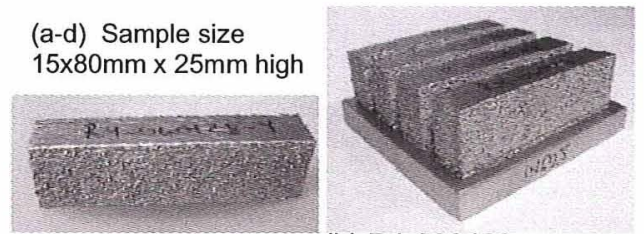

(a) R4-051207

(b) R4-060428
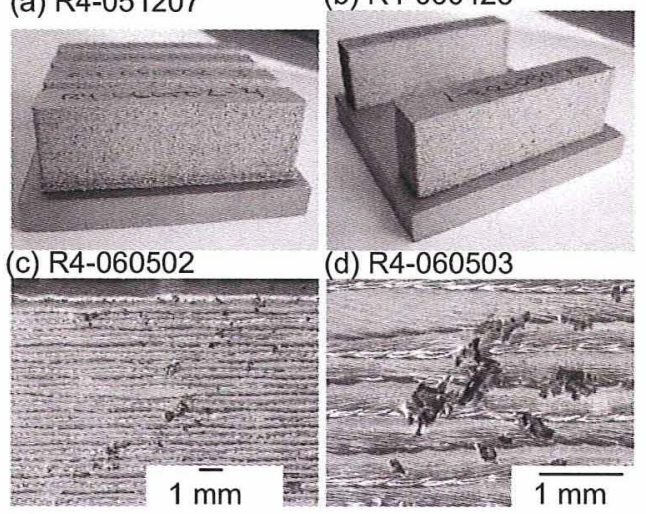

(d) R4-060503

(e) R4-051207

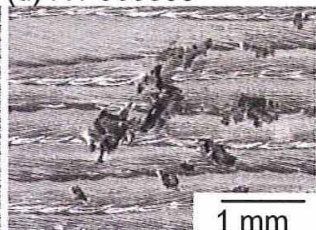

(f) R4-051207

FIGURE 5. EBM Fabricated Test Samples.

The results of the Brinell hardness test are shown below. The results include the wrought annealed Inconel 625 hardness as documented in the ASM Handbook, $10^{\text {th }}$ edition. The measurements were taken at various positions along the build direction $(\mathrm{Z})$.

TABLE 3. Brinell Hardness of Built Material

\begin{tabular}{|c|c|c|c|c|}
\hline $\begin{array}{c}\text { Wrought Annealed } \\
\text { IN625 }\end{array}$ & R4-051207 & R4-060428 & R4-060502 & R4-060503 \\
\hline $190 \mathrm{Ksi}$ & $179,176,178 \mathrm{ksi}$ & $199,202,200 \mathrm{ksi}$ & $202,201,200 \mathrm{ksi}$ & $193,192,192 \mathrm{ksi}$ \\
\hline
\end{tabular}

The tensile tests reflect material strengths that fall between cast and wrought Inconel 625. Optimization of the build process and the addition of post-treatments can provide improved strength characteristics. The test results are shown below.

TABLE 4. Tensile Properties of Built Material

\begin{tabular}{|l|l|l|l|l|l|c|}
\hline $\begin{array}{l}\text { Material } \\
\text { Properties }\end{array}$ & $\begin{array}{l}\text { Wrought } \\
\text { Annealed } \\
\text { IN625 }\end{array}$ & $\begin{array}{l}\text { Polycrystalline } \\
\text { cast IN625 }\end{array}$ & R4-051207 & R4-060428 & R4-060502 & R4-060503 \\
\hline UTS, MPa (RT) & 930 & 710 & 702,673 & 733,780 & 784,789 & 746 \\
\hline YTS, MPa (RT) & 517 & 350 & 349,355 & 423,424 & 426,430 & 395 \\
\hline $\begin{array}{l}\text { Elongation, } \\
\text { (RT) }\end{array}$ & 42.5 & 48 & $34.5,27$ & $41.5,44.5$ & $38.5,40$ & 44.5 \\
\hline (RT) & Not specified & Not specified & 37,28 & 59,66 & 41,66 & 61 \\
\hline
\end{tabular}


At the conclusion of the Inconel 625 feasibility study, it was determined that the material can be used in the machine based on the promising results. The powder melts very well and the process was shown stable after hundreds of melted layers. After initial smoking in the chamber, the material does not smoke easily. The initial smoking can be minimized but not totally eliminated by an extra preheat of the powder surface.

\section{CONCLUSIONS}

The Fabrication Technologies research team selected an additive metal-fabrication system after performing a trade study assessment of layered fabrication technologies. Targeted materials, representative of typical space flight hardware requirements, were identified using historical parts failure data and material-usage analyses. A trade study was then performed to assess part quality and material-set capabilities that are currently existing or anticipated to occur in the near future. Based on the trade study factors, it was concluded that the EBM process was the best suited to the capability requirements of the ISFR team with application to future space environments.

An EBM metal fabrication system at MSFC has been purchased and is in operation and will be used for material-set expansion development and fabrication of demonstration parts to support active programs within NASA, such as the CEV, CLV, and ECLSS, among others. Post-processing techniques will be utilized to improve the fabricated part quality, including CNC machining. The Fabrication Technology team will continue to develop the EBM technology and look for opportunities within NASA/MSFC and other agencies to fulfill the Vision for Space Exploration.

\section{ACKNOWLEDGMENTS}

The authors would like to express their sincere thanks to the colleagues and industry contributors who provided information that made this research possible. Much information was gathered from system vendors and users who are experts in their respective fields. We would also like to thank Arcam AB of Sweden for providing research data as well as photographs as input to this publication.

\section{REFERENCES}

Hammond, M.S., Good, J.S., Gilley, S.D., and Howard, R.W., "Developing Fabrication Technologies to Provide On-Demand Manufacturing for Exploration of the Moon and Mars," $44^{\text {th }}$ AIAA Aerospace Sciences Meeting and Exhibit, Reno, NV, 2006.

Lindhe, U., Harrysson, O., "Rapid Manufacturing with Electron Beam Melting (EBM) - A Manufacturing Revolution?" Society' of Manufacturing Engineers Technical Paper, August 2003.

Bodiford, M.P., Gilley, S.D., Howard, R.W., Kennedy, J.P., Ray, J.A., “Are We There Yet?...Developing In Situ Fabrication and Repair (ISFR) Technologies to Explore and Live on the Moon and Mars" $1^{\text {st }}$ Space Exploration Conference: Continuing the Voyage of Discovery, Orlando, Florida, 2005.

Cooper, K.G., "Extending Rapid Prototyping Past the Horizon: Applications in Outer Space", Society of Manufacturing Engineers Technical Paper, Feb. 2002.

Cooper, K.G., "Microgravity Manufacturing Via Rapid Prototyping Techniques", Georgia Tech Rapid Prototyping and Manufacturing Symposium, May 2001. 